\title{
COVID-19: REFLEXÃO DA ATUAÇÃO DO ENFERMEIRO NO COMBATE AO DESCONHECIDO
}

Júlio César Rabêlo Alves ${ }^{1}$ Mayana Bonfim Ferreira² https://orcid.org/0000-0002-4067-0215

https://orcid.org/0000-0002-1160-1552

Objetivo: Refletir sobre as consequências da atuação do enfermeiro perante o surgimento da COVID-19. Método: Trata-se de um artigo de reflexão, com a coleta de dados entre março e abril de 2020, por meio de bases científicas como a Biblioteca Nacional de Medicina, Journal of the American Medical Association e Scientific Eletronic Library Online. Foram selecionados 10 artigos, nos idiomas em português e inglês, com limitação devido a abordagem recente. Resultados: Torna-se evidente que o novo coronavírus está sendo o maior desafio enfrentado pelo mundo, com uma rápida disseminação. O uso de máscaras, uma boa higiene das mãos e a descontaminação da superficie são fundamentais para a segurança. Entretanto, há uma limitação da quantidade de equipamentos de proteção individual, somado a sobrecarga emocional dos enfermeiros e as péssimas condições de trabalho que já os acompanham, eles que estão na linha de frente no combate. Em meio ao desconhecido, uma estratégia especial para a atuação da enfermagem é necessária, protegendo-os. Conclusão: À COVID-19 envolve diversos fatores da sociedade, e gera muitas incertezas. No epicentro dessa catástrofe estão os enfermeiros, que em meio as tamanhas adversidades vêm demonstrando ainda mais as suas competências. Por isso, é importante informações precisas e uma valorização profissional para que nesse combate o bem-estar fique fortalecido.

Descritores: Enfermagem; Coronavirus; Infecções Respiratórias.

\section{COVID-19: REFLECTION ON THE ROLE OF NURSES IN COMBATING THE UNKNOWN}

Objective: To reflect the consequences of nurses' actions in the face of the emergence of COVID-19. Method: This is a reflection article, with data collection between March and April 2020, through scientific databases such as National Library of Medicine, Journal of the American Medical Association and Scientific Eletronic Library Online. 10 articles were selected in Portuguese and English languages, with limitation due to the recent approach. Results: It becomes evident that the new coronavirus is being the biggest challenge faced by the world, with a rapid spread. The use of masks, good hand hygiene and surface decontamination are essential for safety. However, there is a limitation of the amount of personal protective equipment, added to the emotional overload of nurses and the poor working conditions that already accompany them, who are on the front line in combat. In the midst of the unknown, a special strategy for nursing practice is necessary, protecting them. Conclusion: COVID-19 involves several factors of society, and generates many uncertainties. At the epicenter of this catastrophe are nurses, who in the midst of such adversities have been demonstrating even more their skills. Therefore, it is important to accurate information and a professional appreciation so that in this fight the well-being is strengthened.

Descriptors: Nursing; Coronavirus; Respiratory infections.

\section{COVID-19: REFLECCION SOBRE EL ROLE DE LAS ENFERMERAS EN EL COMBATE DE LO DESCONOCIDO}

Objetivo: Reflejar las consecuencias de las acciones de los enfermeros frente a la aparición da COVID-19. Metodo: Este es un artículo de reflexión, con recopilación de datos entre marzo y abril de 2020, a través de bases de datos científicas como Biblioteca Nacional de Medicina, Journal of the American Medical Association y Scientific Eletronic Library Online. 10 artículos fueron seleccionados en portugués e inglés, con limitación debido al reciente enfoque. Resultados: Se hace evidente que el nuevo coronavirus está siendo el mayor desafio al que se enfrenta el mundo, con una rápida propagación. El uso de mascarillas, una buena higiene de las manos y la descontaminación de la superficie son esenciales para la seguridad. Sin embargo, hay una limitación de la cantidad de equipo de protección personal, sumado a la sobrecarga emocional de las enfermeras y a las malas condiciones de trabajo que ya los acompañan, que están en primera línea en combate. En medio de lo desconocido, es necesaria una estrategia especial para la práctica de enfermería, protegiéndolas. Conclusión: COVID-19 implica varios factores de la sociedad, y genera muchas incertidumbres. En el epicentro de esta catástrofe están las enfermeras, que en medio de tales adversidades han estado demostrando aún más sus habilidades. Por lo tanto, es importante obtener información precisa y un aprecio profesional para que en esta lucha se fortalezca el bienestar.

Descriptores: Enfermería; Coronavirus; Infecciones respiratorias.

${ }^{1}$ Fundação Estatal de Saúde da Familia FESF-SUS/ FIOCRUZ, Pojuca, BA

${ }^{2}$ Centro Universitário Jorge Amado - UniJorge, BA.

Autor Correspondente: Júlio César Rabêlo Alves. E-mail: papito.julio@hotmail.com Recebido: 28/4/2020 - Aceito: 26/5/2020 


\section{INTRODUÇÃO}

A COVID-19 integra uma das doenças causadas pelo coronavírus, este que forma uma família de vírus que causam infecções respiratórias, e tem um histórico de acometimentos desde o século passado. Já o novo agente patológico foi descoberto no dia 31/12/2019, na China, após crescentes casos de pneumonia. Seu poder de contaminação é tão rápido que o tempo entre a sua manifestação inicial e a declaração de pandemia pela Organização Mundial de Saúde (OMS) foram de apenas 3 meses ${ }^{(1)}$.

Muitos se questionam o seu surgimento e especulam diversas teorias que vão desde a econômica, criação em laboratório ou zoonoses. Em meio a tantas incertezas que essa crise apresenta, é certo que a doença se torna mais letal nos idosos e doentes crônicos, especificamente para os Diabéticos e Hipertensos. E o principal sintoma apresentado é a febre, seguida de tosse seca e/ou dificuldade para respirar, este último que caracteriza a forma mais grave da enfermidade ${ }^{(2)}$. Nesse cenário, o Sistema de Saúde foi desafiado, o que se tem de informação é que países que foram severamente acometidos estão com a taxa de ocupação dos leitos de UTI completamente lotados ${ }^{(3)}$.

Diante dessa realidade, o Brasil vem tomando medidas de contenção, como na criação de Hospitais de Campanha, para aumentar a demanda de leitos, prevendo o que de pior pode acontecer. Na linha de frente temos os profissionais de saúde, em especial atenção a enfermagem, que é considerada uma espinha dorsal, segundo relatório publicado pela $\mathrm{OMS}^{(4)}$.

Nesse cenário, houve uma elevação dos números de casos suspeitos e confirmados da COVID-19 pelos enfermeiros. Estes que juraram dedicar a vida profissional a serviço da humanidade, com responsabilidade, dedicação e conhecimento técnico-científico no cuidado e tratamento dos pacientes. E é o que sem tem visto na sua atuação diante desta crise mundial, entretanto, há um excesso de carga horária de trabalho, escassez dos equipamentos de proteção individual (EPI) e salários injustos, que causam um estresse ocupacional. Todos esses fatos elencados que por si só já se fazem presente no dia a dia destes profissionais ${ }^{(5)}$, e com a chegada desse novo virus ficou ainda mais evidente essa precariedade trabalhista.

Com essa analogia, a falta de medicamentos específicos para a cura e o alto potencial de contaminação e transmissão são as principais causas para o crescimento da taxa de exaustão psíquica entre os profissionais no combate ao coronavírus. Os enfermeiros ficam tão incumbidos em agir e esquecem, muitas vezes, de cuidar de si mesmo(b)
Baseado nisso, questionamos: Quais os reflexos que podem acometer os enfermeiros no combate ao desconhecido? E quais os desafios enfrentados diante desse cenário? E para responder tais questões, este artigo tem como objetivo refletir as consequências da atuação do enfermeiro perante o surgimento da COVID-19.

\section{MÉTODO}

Trata-se de um artigo de reflexão, com o propósito de debater sobre o assunto abordado e aprimorar os conhecimentos sobre tal. Pois a partir do momento atual, uma nova pandemia, procura-se refletir sobre a importância do enfermeiro no combate a esse desconhecido. A coleta de dados ocorreu entre março e abril de 2020, por meio da busca de artigos científicos nas bases de dados Biblioteca Nacional de Medicina (PubMed), Journal of the American Medical Association (JAMA) e Scientific Eletronic Library Online (SciELO).

Na realização deste estudo foi definido os descritores: "Enfermagem"; "Coronavírus"; “Infecções Respiratórias", de acordo com a classificação dos Descritores em Ciências de Saúde (DESC). Foram selecionados 10 artigos, sendo filtrados pelos seguintes critérios de inclusão: leitura na íntegra, gratuitos e recorte dos últimos 5 anos em português e inglês. Como critérios de exclusão: artigos incompletos, duplicados nas bases e que não contemplassem o tema abordado. É importante ressaltar a limitação de conteúdos disponíveis, principalmente na língua portuguesa, por ser uma temática de estudo muito recente.

A análise dos artigos foi realizada diante de uma leitura exploratória e validados os que correspondiam ao propósito da pesquisa, respondendo ao conjunto de questionamentos impostos para a reflexão. Com as ideias baseadas nas situações de trabalho dos enfermeiros no atual cenário de pandemia e as suas principais dificuldades enfrentadas, fazendo uma correlação com os impasses que já eram vivenciados antes deste acometimento.

\section{RESULTADOS}

Diante dos artigos lidos e do que é noticiado diariamente, torna-se evidente que à COVID-19 é o maior desafio enfrentado pelo mundo nesse milênio. Imaginou-se que uma terceira guerra mundial fosse constituída de avançados armamentos nucleares, mas ninguém previa que seria por algo invisível e difícil de controlar. Esta que é o principal desafio para os profissionais de saúde, em limitar ao máximo a contaminação em massa.

No entanto, a característica desse vírus faz com que haja uma grande taxa de pessoas assintomáticas, e essas 
são as principais disseminadoras. Então, o uso de máscaras e outras precauções de barreira, a higiene das mãos aprimorada e a descontaminação da superfície são fundamentais para a segurança ${ }^{(7)}$. Sendo assim, o método mais eficaz é a educação permanente como medida preventiva, e nada melhor do que a Enfermagem, que naturalmente já desempenha esse papel. Essa que é a medida mais importante na ausência de uma vacina e medicamento específico para este virus.

Em meio ao desconhecido, as atualizações diárias das notícias são importantes, principalmente vindas de estudos dos países que estão em uma etapa mais avançada da doença. Com isso, o papel da mídia em transmitir informações precisas e fidedignas, em tempos de "fake news", é fundamental. E o que se tem noticiado são o grande risco que os profissionais de saúde correm, com crescente indices de contaminação entre eles.

Esse fato se justifica pela escassez dos Equipamentos de Proteção Individual (EPI) e a falta de qualificação no uso dos mesmos. Já que o novo coronavírus necessita das precauções recomendadas de barreira, como máscaras cirúrgicas / N95, luvas, aventais e óculos de proteção ou protetores faciais, no cuidado de todos os pacientes assintomáticos ou apresentando sintomas respiratórios, que indica alta prioridade do uso dos $\operatorname{mesmos}^{(7)}$

Além disso, a sobrecarga emocional pode justificar o aumento da contaminação entre os profissionais de saúde, levando a problemas na Saúde Mental dos enfermeiros, que desencadeiam nos estresses, diminuição da atenção, medo e preocupações demasiadas devido ao desconhecido. Eles que estão na linha de frente do cuidado durante a pandemia, com isso, acaba ocasionando o estresse ocupacional. Esta que tem como característica o esforço físico para se adequar aos diferentes acontecimentos diários que lhe são postas, consequências das relações entre demanda psicológica e controle, associado ao processo da patologia ${ }^{(8)}$.

\section{DISCUSSÃO}

Podemos afirmar que estes desafios elencados são capazes de estimular comportamentos e ações nos profissionais de saúde, como nas relações estressantes existentes entre os trabalhadores e seu ambiente de trabalho, podendo colaborar para predisposição até o desenvolvimento de doenças. E é o que se tem visto na COVID-19, somado a isto, é importante elencar as precárias condições de trabalho que a enfermagem enfrenta há anos. A carga horária extensiva, os salários injustos e a ausência do piso salarial tornam-se mais evidentes nesse cenário de crise da saúde brasileira.
Diante dessa realidade, os enfermeiros estão em uma situação de vulnerabilidade. O grande tempo de exposição trabalhista e o rápido crescimento de pacientes contaminado pelo novo coronavírus potencializa todos os fatores citados. Expor seus familiares a esta situação também é uma problemática a ser enfrentada, e o que se tem visto são que eles estão abdicando da sua vida familiar durante esse período incerto, para evitar a possivel contaminação dos seus entes queridos. Os profissionais de saúde desejam uma garantia inequívoca de que sua organização apoiará eles e sua família( ${ }^{(1)}$.

Por outro lado, muitas vezes, este fato não acontece. A segurança esperada pelos trabalhadores não se reflete na realidade, que está pautada em fatores financeiros que se sobrepõe a atividade humana, no pensamento institucional. Qual o limite e consequência deste raciocínio? O limite não é possivel ponderar, mas as consequências já estão sendo sentidas pelo despreparo da organização em saúde, que já era evidente e tratada com descaço, mas que agora é mais do que visivel.

Assim, é importante traçar uma estratégia especial para a atuação da enfermagem, protegendo-os, como por exemplo: na diminuição da sobrecarga profissional, nos constantes treinamentos com base nas atualizações publicadas, oferta de todos EPI e em quantidade suficiente, disponibilização de atendimento psicológico e na valorização profissional. Medidas essas que precisam permanecer depois que essa situação amenizar, mais do que isso, precisam ser intensificadas. Pois uma enfermagem fortalecida reflete em um melhor sistema de saúde.

Por essa razão, é necessário ressaltar que as ações de isolamento social adotadas, por si só, não eliminam o vírus. Elas servem mais como parâmetro de ganhar tempo para o fortalecimento e melhor preparo dos serviços de saúde. Com isso, os órgãos governamentais precisam dar os suportes essenciais para que os enfermeiros estejam preparados para atender a demanda populacional mais desfavorecida e manter o equilibrio emocional.

Bem como, é importante lembrar que em meio a essa pandemia, os demais problemas de saúde permanecem, assim como os atendimentos para eles. Diante dessa situação uma boa estratégia seria o bom uso da tecnologia, evitando um colapso ${ }^{11}$. Mas nada substitui o contato presencial, papel que a enfermagem desempenha no cuidado e que não pode, muitas vezes, ser substituido. O certo é que, tomar decisões e fazer escolhas nessas condições, do desconhecido e de incertezas, é um enorme desafio, e a chance de errar é grande ${ }^{\left({ }^{2}\right)}$ 


\section{CONSIDERAÇÕES FINAIS}

A COVID-19 é uma doença cruel, e a cada dia testa a capacidade do sistema de saúde em lidar com os problemas derivados desse vírus. Mais do que isso, envolve fatores sociais e econômicos, que geram incertezas diárias nas condutas que se deve tomar. No epicentro dessa catástrofe estão os enfermeiros, atuando em uma diversidade de setores, deste a atuação direta ao novo vírus ou áreas associadas. Estes que em meio a vários desafios vem demonstrando suas competências, assim como sempre fazem no seu dia a dia, e agora tornou-se mais evidente para o contingente populacional. Logo, é necessário refletir melhorias na exaustiva atuação dos enfermeiros frente a esse desconhecido, que assusta e os coloca em extrema situa- ção adversa, como na falta de EPI e no comprometimento da sua saúde mental, mas mesmo assim eles não param. Por isso, é importante, mais do que nunca, dispor de informações precisas e considerar uma valorização profissional, para que nesse combate o bem-estar fique fortalecido.

\section{CONTRIBUIÇÕES DOS AUTORES}

Júlio César Rabêlo Alves contribuiu para a concepção e desenho do estudo, análise e interpretação dos dados, redação e revisão crítica do manuscrito, e aprovação da versão final a ser publicada; Mayana Bonfim Ferreira concepção e desenho do estudo; coleta e análise dos dados, revisão crítica do manuscrito e aprovação da versão final a ser publicada.

\section{REFERÊNCIAS}

1. Organização Pan-Americana da Saúde (OPAS). Folha informativa - COVID-19 (doença causada pelo novo coronavirus). Brasilia - DF: OPAS: 2020 [citado em 20 de maio de 2020]. Disponivel em: https:// www.paho.org/bra/index.php?option=com_content\&view=articleEi$d=6101$ :COVID198Itemid $=875$

2. Wang D. Hu B, Hu C, Zhu F, Liu X, Zhang J, et al. Clinical Characteristics of 138 Hospitalized Patients With 2019 Novel Coronavirus-Infected Pneumonia in Wuhan, China. JAMA. 7 de fevereiro de 2020 [citado 18 de abril de 2020]; 323 (11): 1061-1069. Disponivel em: https://jamanetwork.com/journals/jama/article-abstract/2761044

3. Moreira RS. COVID-19: unidades de terapia intensiva, ventiladores mecânicos e perfis latentes de mortalidade associados à letalidade no Brasil. Rio de Janeiro: Cadernos de Saúde Pública. 18 de maio de 2020 [citado 22 de maio de 2020]: 36(5):e00080020. Disponivel em: https:// www.scielo.br/pdf/csp/v36n5/1678-4464-csp-36-05-e00080020.pdf

4. World Health Organization (WHO). State of the world's nursing 2020: investing in education, jobs and leadership. Geneva: World Health Organization; 6 de abril de 2020 [citado em 19 de abril de 2020]. Licence: CC BY-NC-SA 3.0 IGO. Disponivel em: <https://www.who.int/publications-detail/nursing-report-2020>

5. Ribeiro RP, Marziale MHP, Martins JT, Galdino MJQ, Ribeiro PHV. Estresse ocupacional entre trabalhadores de saúde de um hospital universitário. Revista Gaúcha de Enfermagem. 23 de julho de 2018 [citato 20 de maio de 2020]; 39:e65127. Disponivel em: https://www.scielo.br/scielo. php?pid=S1983-14472018000100421\&script=sci_arttext\&tlng=pt

6. Rolim Neto ML, Almeida HG, Esmeraldo JD, Nobre CB, Pinheiro WR, Oliveira CRT et al. When health professionals look death in the eye: the mental health of professionals who deal daily with the 2019 coronavirus outbreak. Psychiatry Research. 13 de abril 2020 [citado 21 de abril de 2020]: 288 (112972). Epub junho de 2020. Disponivel em: https://pubmed.ncbi.nlm.nih.gov/32302817/

7. Adams JG, Walls RM. Supporting the Health Care Workforce During the COVID-19 Global Epidemic. JAMA. 12 de março de 2020 [ci-

tato 21 de abril de 2020]: 323(15):1439-1440. Disponivel em: https:// jamanetwork.com/journals/jama/fullarticle/2763136?guestAccessKey=3bb66b23-edb9-40b9-92105016ba70da898utm_source=For_ The_Media\&utm_medium=referral\&utm_campaign=ftm_links\&utm_ content=tfl\&utm_term $=031220$

8. Vilas-Boas M, Cerqueira A. Avaliação do estresse no trabalho: a versão portuguesa do Job Content Questionnaire. Avaliação Psicológica. 2017 [citado 22 de abril]; 16(1):70-77. Disponivel em: http://pepsic.bvsalud.org/scielo.php?script=sci_arttext\&pid=S1677-04712017000100009

9. Cavalcante JL, Pinto AGA, Brito Júnior FE, Moreira MRC, Lopes MSV, Cavalcante EGR. Estresse ocupacional dos funcionários de uma universidade pública. Revista Enfermagem em Foco, 2019 [citato 22 de abril de 2020]; 10(4):108-115. Disponivel em http://revista.cofen.gov. br/index.php/enfermagem/article/view/2310/612.

10. Shanafelt T, Ripp J. Trockel M. Understanding and Addressing Sources of Anxiety Among Health Care Professionals During the COVID-19 Pandemic. JAMA. 7 de abril de 2020 [citado 22 de abril de 2020]; doi: 10.1001 / jama.2020.5893. Disponivel em: https://jamanetwork.com/journals/jama/fullarticle/2764380?widget=personalizedcontentEpreviousarticle=2763136

11. Teich N. COVID-19, Telemedicina e Eficiência do Sistema de Saúde. LinkedIn. 18 de março de 2020 [citato 24 de abril de 2020]. Disponivel em: https://www.linkedin.com/pulse/COVID-19-telemedicina-e-efici\%C3\%AAncia-do-sistema-de-sa\%C3\%BAde-nelson-teich?articleId=6646115550886010880\#comments-6646115550886010880\&trk=public_profile_article_view

12. Teich N. COVID-19: Histeria ou Sabedoria? LinkedIn. 24 de março de 2020 [citato 24 de abril de 2020]. Disponivel em: https://www.linkedin.com/pulse/COVID=19--histeria-ou-sabedoria-nelson-teich?articleId6648224510938013696\#comments-66482245109380136968trk=public_profile_article_view 